\title{
Effect of Sting Geometry on Axial Force Calculation for the Space Launch System
}

\author{
Christopher A. Eggert*, Patrick R. Shea, Ph.D. ${ }^{\dagger}$, Nalin A. Ratnayake ${ }^{\ddagger}$, and Steven E. Krist, Ph.D. ${ }^{\S}$ \\ NASA Langley Research Center, Hampton, VA, 23681-2199
}

The primary purpose of this study is to determine the extent to which the size and shape of the wind tunnel sting affect the accuracy of the base pressure corrections applied to measured axial force. The study also includes an assessment of the overall accuracy of the corrections. To accomplish these goals, Computational Fluid Dynamics is used to simulate a simplified version of the geometry of the Space Launch System Block 1B Cargo configuration, paired with a range of wind tunnel sting sizes, over a variety of ascent flight conditions. The base pressure correction method used in the wind tunnel is emulated on the base pressures from the simulated flows and results are compared to direct integration of the base pressures. Differences in results between the two methods provides an assessment of the accuracy of the base force correction method and how that accuracy is affected by sting size.

\section{Nomenclature}

$\begin{array}{ll}C_{A} & =\text { Axial Force Coefficient }(C A \text { on figure axis labels) } \\ C_{P} & =\text { Pressure Coefficient }(C P \text { on figure axis labels }) \\ M & =\text { Mach Number } \\ \alpha & =\text { Pitch Angle }[\mathrm{deg}] \\ \beta & =\text { Sideslip Angle }[\mathrm{deg}] \\ A U P W T & =\text { Ames Unitary Plan Wind Tunnel } \\ C F D & =\text { Computational Fluid Dynamics } \\ D D E S & =\text { Delayed Detached Eddy Simulation } \\ D E S & =\text { Detached Eddy Simulation } \\ L E S & =\text { Large Eddy Simulation } \\ N B O B & =\text { Near-Body, Off-Body } \\ O M L & =\text { Outer Mold Line } \\ R A N S & =\text { Reynolds-Averaged Navier-Stokes } \\ S L S & =\text { Space Launch System } \\ S R B & =\text { Solid Rocket Booster (Right }- \text { RSRB; Left }- \text { LSRB) }\end{array}$

\section{Introduction}

$\mathrm{O}$ NE challenge in obtaining accurate values for the axial force acting on a launch vehicle like the Space Launch System (SLS) lies in how the pressure on the base region of the vehicle is accounted for during wind tunnel tests. During full-scale flights of the vehicle, the airflow in this region will be significantly affected by the exhaust plumes of the rocket motors. However, since these plumes are generally not present during wind tunnel testing and the model support hardware can influence the pressures at the base of the vehicle, base pressure corrections are applied to subtract the forces and moments acting on the exhaust-affected parts of the vehicle from the total measured force values. Plume and engine-on effects are typically added later as an increment to the aerodynamic databases used for structural analysis and the development of trajectories for the purposes of Guidance, Navigation, and Control.

Generally, base pressure is approximated by averaging a small number of pressure tap readings taken on the base of

\footnotetext{
*Aerodynamics Intern, Configuration Aerodynamics Branch, AIAA Student Member

'Research Aerospace Engineer, Configuration Aerodynamics Branch, AIAA Member

${ }^{\ddagger}$ Research Aerospace Engineer, Configuration Aerodynamics Branch, AIAA Senior Member

${ }^{\S}$ Research Aerospace Engineer, Configuration Aerodynamics Branch, AIAA Senior Member
} 
the vehicle. These values are then multiplied by the area of that body's base region and the resultant force is subtracted from the force measured by the load balance, to produce the corrected axial force. For the SLS, the correction involves taking pressures at the base of each of the three bodies, namely, the two solid rocket boosters (SRBs) and the core. For a large program like that of the SLS, several different wind tunnels, wind tunnel models, and sting geometries may be used over the duration of many development cycles [1-4]. Slight variations in factors like pressure tap location and base cavity shape are also common between different wind tunnel tests. These differences can make direct comparison of the resultant data difficult, since it is unknown how they interact with the correction method to impact the accuracy of the reported data.

The primary purpose of this study is to determine the extent to which the size and shape of the wind tunnel sting affects the accuracy of the base pressure corrections applied to the measured axial force. The three-body geometry of the SLS vehicle adds interference and flow effects not present in single-body launch vehicle configurations, and wind tunnel support interference has not been studied as extensively for multibody geometries as for simple bodies of revolution (see, for example, Refs. [5-8]). To the extent possible, the current study is also intended to provide an assessment of the overall accuracy of the current base pressure correction method. To accomplish these goals, Computational Fluid Dynamics (CFD) simulations are used to assess the base forces on a simplified version of the Space Launch System Block 1B Cargo configuration, paired with a range of wind tunnel sting sizes, over a variety of ascent flight conditions. Results from an emulation of the pressure correction method applied to the simulated flows are compared to results from direct force integration on the desired surfaces. By observing the difference between these two methods, the accuracy of the pressure correction method can be assessed as a function of sting size. The results of this study are intended to be used to inform best practices for future axial force measurement and prediction for the SLS and similar three-body launch vehicles.

\section{Methods}

\section{A. Geometry Description}

As shown in Fig. 1, the configuration used for this study was derived from a highly simplified SLS geometry. In order to minimize the size of the grid, the least complex, available SLS nose shape was selected, all rings and protuberances were eliminated from the model, and the forward and aft SRB attach brackets were simplified. These modifications reduce the computational cost of each CFD simulation. However, those savings were offset somewhat by the need to highly refine the separated wake region at the base of the vehicle. This geometry was also symmetric across

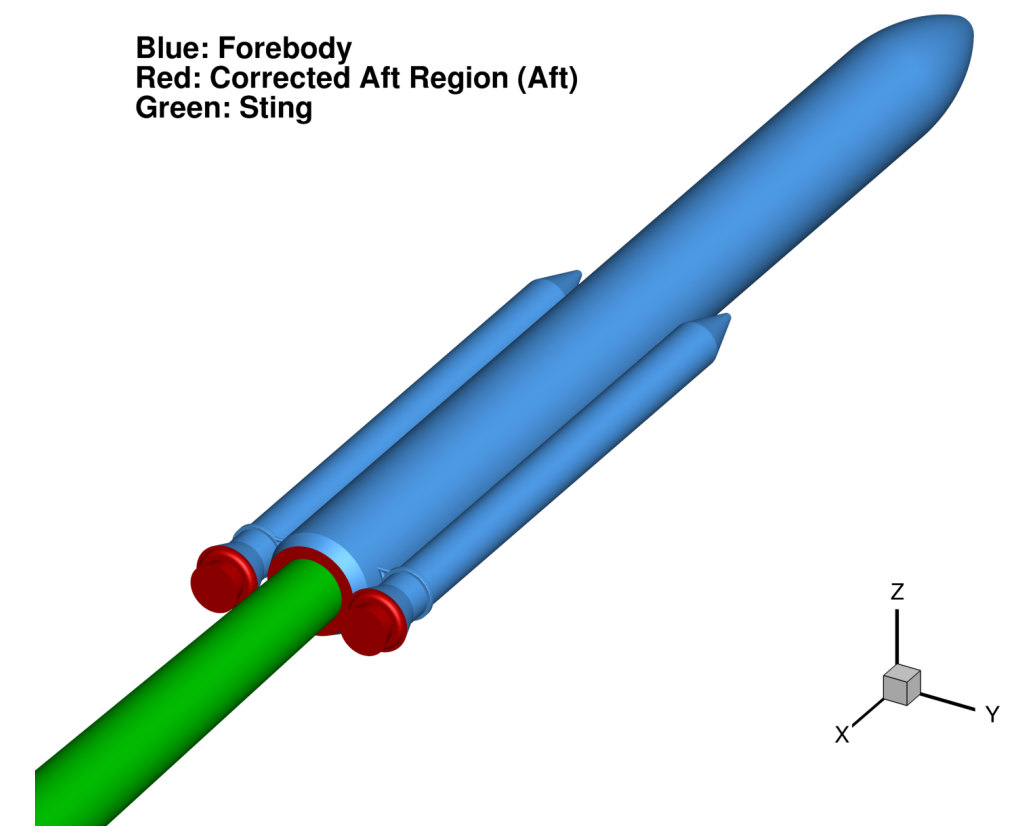

Fig. 1 Simplified SLS Block 1B Cargo configuration used for CFD base pressure assessment. 
the XZ plane, and nearly symmetric across the XY plane as well (the aft SRB attach brackets are slightly asymmetric).

A major difference between the CFD geometry and the wind tunnel model is the lack of a base cavity. The SLS wind tunnel models used for force and moment testing are hollow and open at the base to allow the model to deflect on the force balance when the model is under load without contacting the sting. It is known that this cavity plays a major role in determining the pressure distribution at the base of the core. Unfortunately, CFD solvers for the compressible Reynolds-Averaged Navier-Stokes (RANS) equations are not particularly well suited for accurately reproducing the characteristics of the large region of recirculating, low-velocity, internal flow present inside of a hollow model. Moreover, the grid resolution requirements for such an attempt are as stringent as those for the wake behind the base. Therefore, in the interest of minimizing the grid size and complexity for this study, the decision was made to close off the base of the model with a flat surface on both the core and the two SRBs in the CFD models, leaving inclusion of the cavity in the CFD models for subsequent investigation. While this may compromise the comparison between the CFD-calculated base pressures to those measured in the wind tunnel, accurate comparison between the CFD solutions themselves should remain possible. In other words, the pressure profile on the core base surface and SRB nozzle base surfaces modeled in CFD should provide a useful plane on which to study how much the sting size affects the flow field in this region.

Six different sting geometries were created to be paired with the vehicle. First, the shape of the wind tunnel sting used in experiments at the Ames Unitary Plan Wind Tunnel (AUPWT) was replicated. This sting has an area ratio (the ratio between the cross-sectional area of the sting and the area of the base of the core) of 39\%, and is tapered in near the model. Then, a series of four cylindrical stings was created. These stings had area ratios of $5 \%, 10 \%, 20 \%$, and $40 \%$. Finally, the vehicle was tested with no sting attached (an area ratio of $0 \%$ ). In the 3D models used for CFD, the stings make direct contact with the base of the core and extend all the way to the back of the flow domain, intersecting the boundary. A comparison of the six geometries can be seen in Fig. 2. Using the full set of stings, a so-called "sting sweep" could be performed, in which all six sting geometries were run at the same flow conditions to check trends in forces against sting area ratio. It should be noted that, whenever comparisons are shown against wind tunnel data, the wind tunnel data were only taken with the AUPWT sting, and with the model cavity not simulated in CFD.

Area Ratio: 0\% (NoSting)

Area Ratio: 5\% (ar05)

Area Ratio: $10 \%$ (ar10)

Area Ratio: $20 \%$ (ar20)

Area Ratio: $40 \%$ (ar40)

AUPWT
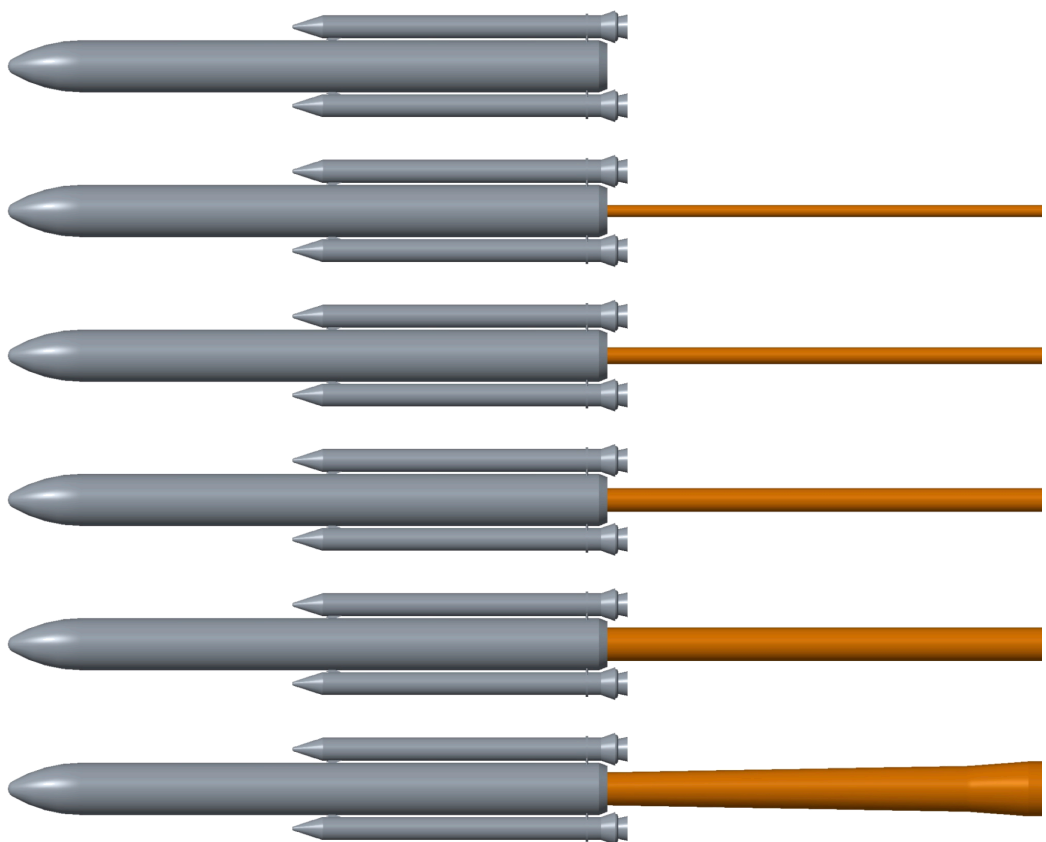

Fig. 2 Six configurations were studied; no sting, four cylindrical stings of increasing area ratio, and the AUPWT sting. 


\section{B. Wind Tunnel Methods}

Since the purpose of this study is to examine wind tunnel methodology, a brief overview of the objectives and methods of experimental work on the SLS is necessary. In the case of the ascent tests, which take place between Mach 0.5 and 5.0, the purpose of the wind tunnel experiments is to gather six degree-of-freedom force and moment coefficients on the forebody of the SLS (a diagram is provided in Fig. 1 that shows what parts of the SLS model are included in the forebody). Since the raw measured force values on the model will also include the forces on the base (or aft) region, the experimentalists must apply a correction before reporting the forebody axial force.

As shown in Fig. 3 for the SLS ascent experiments, the correction was implemented by using six pressure probes near the base of the model: one at the center of the base of each SRB and four located behind the core in a ring around the wind tunnel sting, offset $90^{\circ}$ from each other. The pressure coefficients obtained by these probes were then multiplied by the rear-facing area of their respective components. In the case of the four probes on the SLS core, the pressure coefficient values were averaged before being multiplied by the core base area. In the case of the SRB probes, the measured pressure coefficients were simply multiplied by the SRB base area. The summation of the axial force corrections on the three bodies was then subtracted from the axial force measured by the wind tunnel balance to obtain the forebody axial force.

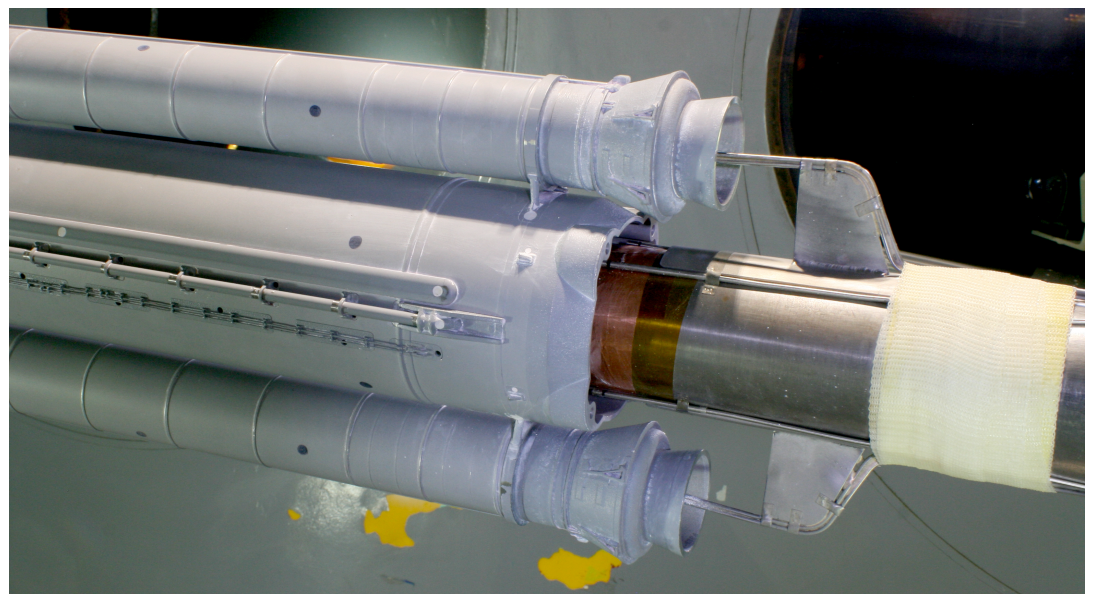

Fig. 3 Example of typical SLS base pressure installation [3].

Since the pressure on the base of the model is approximated using only these six pressure probes, it is apparent that the uniformity of the pressure on the base of the model will determine how accurate the corrections are. To examine the effectiveness of the six-probe method, an emulation script was created that could perform the same approximations from the wind tunnel methods on a CFD solution. The emulator probes the CFD solution flow field at the same locations that the pressure taps would be placed on the wind tunnel model and calculates the base force correction in the same way. For the cases in which no sting geometry is included, the four core probes are all located at the center of the base of the core. When this emulator is used, a direct comparison can be made between forces obtained through direct force integration of a CFD solution and the wind tunnel correction method on the same solution. If the direct force integration method is considered to produce the "true" forces acting on the model, then the correction error between the two methods can be quantified by simple subtraction.

\section{CFD Flow Solvers}

This study utilizes three different CFD solvers and a variety of input settings in an attempt to identify the procedures required to attain useful levels of accuracy in the CFD solutions. The three flow solvers employed were USM3D, FUN3D, and Kestrel. USM3D and FUN3D are developed and supported out of the NASA Langley Research Center. Kestrel is developed and maintained by the Computational Research and Engineering Acquisition Tools and Environment, Air Vehicles (CREATE-AV) project under the US Department of Defense High-Performance Computing Modernization Program (DoD HPCMP). All three codes are used to solve the RANS equations on unstructured grids, but with distinct approaches.

USM3D is a second-order accurate, cell-centered flow solver [9]. Although it has the capability to run Detached Eddy Simulations (DES), this study only utilized its RANS solver. All solutions from this code were obtained by 
running it in steady-state mode, as unsteady flow features were not expected at the flow conditions simulated. The main turbulence model used was the Spalart-Allmaras (SA) model, and select cases were run using the Menter Shear Stress Transport (SST) model.

FUN3D is a second-order accurate, node-based solver [10]. It is capable of producing flow solutions using both RANS and Delayed Detached Eddy Simulation (DDES). RANS solutions were mainly used for comparison with results obtained from USM3D, so the settings and grid strategy were kept as similar as possible. For both RANS and DDES runs, the main turbulence model used was SA, although a subset of cases were run using SST. The fact that FUN3D is a node-based solver does mean that grids for any given case need to be finer than those used for USM3D if the grid resolution of the solutions is to be considered comparable. The distribution of nodes in the FUN3D grid ideally matches the distribution of cells in the USM3D grid, although some discrepancies, especially in the viscous layer thicknesses, are inevitable.

The DDES capability in FUN3D was used to gain additional accuracy in the solutions, and was eventually used to produce the majority of the solutions for this study. DDES methods essentially combine the use of RANS in the boundary layer of the flow and Large Eddy Simulation (LES) in regions outside the boundary layer. In general, LES methods yield far more accurate solutions than RANS methods for flows with massive separation, but LES is also much more expensive, as the grid resolution in both the boundary layers and the wake must be extremely fine in order to resolve the gradients. DDES provides a good balance between solution accuracy and computational resource requirements, as grid resolution of the boundary layers is no more expensive than for RANS, but the fine resolution required in the wakes increases the grid size far beyond what is required for RANS; consequently, DDES runs are on much finer grids than those used for RANS, particularly behind the base of the vehicle. Since the large eddy content of the wake is inherently unsteady, DDES simulations are only run in time-accurate mode.

The Kestrel software utilizes a Common Scalable Infrastructure to implement a modular approach for connecting the computational tools required to conduct multi-disciplinary assessments of fixed-wing aircraft [11]. Among the many capabilities that the architecture enables is a dual mesh approach, referred to as the near-body, off-body (NBOB) methodology, in which the near-body flow is resolved on an unstructured grid that encapsulates the solid body, and the unstructured grid is immersed in a Cartesian mesh that extends to the far field. The advantage of the NBOB methodology is that most of the work on the finely resolved wake grids can be performed on the Cartesian mesh, as the speed of the Cartesian mesh flow solver is much faster than the speed of the unstructured grid flow solver. However, due to issues with the geometry of the wind tunnel sting, this option could not be used for this study; instead, all Kestrel runs were conducted on the unstructured grids used for USM3D.

The flow solver within Kestrel that is utilized on unstructured grids is KCFD, which takes a cell-centered approach similar to that for USM3D. It is capable of RANS and DDES, both of which were utilized on a subset of cases for comparison to USM3D and FUN3D. For RANS, the SA turbulence model was used. For DDES, both SA and SST were tested.

\section{Grid Generation}

The unstructured grids were created using GridTool and Heldenmesh. The GridTool Cocoa (GTC) is one of the tools provided with the Tetrahedral Unstructured Software System (TetrUSS) suite of tools [12], which is developed and maintained at the NASA Langley Research Center. Heldenmesh is a software suite developed by the Helden Aerospace Corporation for the rapid generation of unstructured grids about complex configurations; it was designed to be compatible with, but a robust extension of, the TetrUSS grid generation tools. The version used in this effort is Heldenmesh version 1.1.

The grid generation process begins with importation of the CAD geometry into GTC, where surface patches covering the entire outer mold line (OML) are defined, then grouped to form components. Of primary importance in generating accurate solutions is the source specification. Since the grid spacing and local density of the cells are related to the strengths and locations of the sources, appropriate placement of the sources by the user refines the grid where it is needed, namely, in regions of high flow field gradients. Line sources with anisotropic stretching are typically employed at the boundaries where components intersect or around features that embody sharp discontinuities in the surface. Volume sources in the shape of a cylinder, cone, or sphere are employed within the perimeter of the larger components of the vehicle. A screen shot highlighting the source specification for the AUPWT sting geometry is shown in Fig. 4. Solid yellow lines indicate line sources and green spheres, cones and cylinders indicate volume sources; note that the pink volume source is merely the source that was active when the screen shot was taken.

In developing grids suitable for the implementation of DDES methods, volume sources must be extended well out 


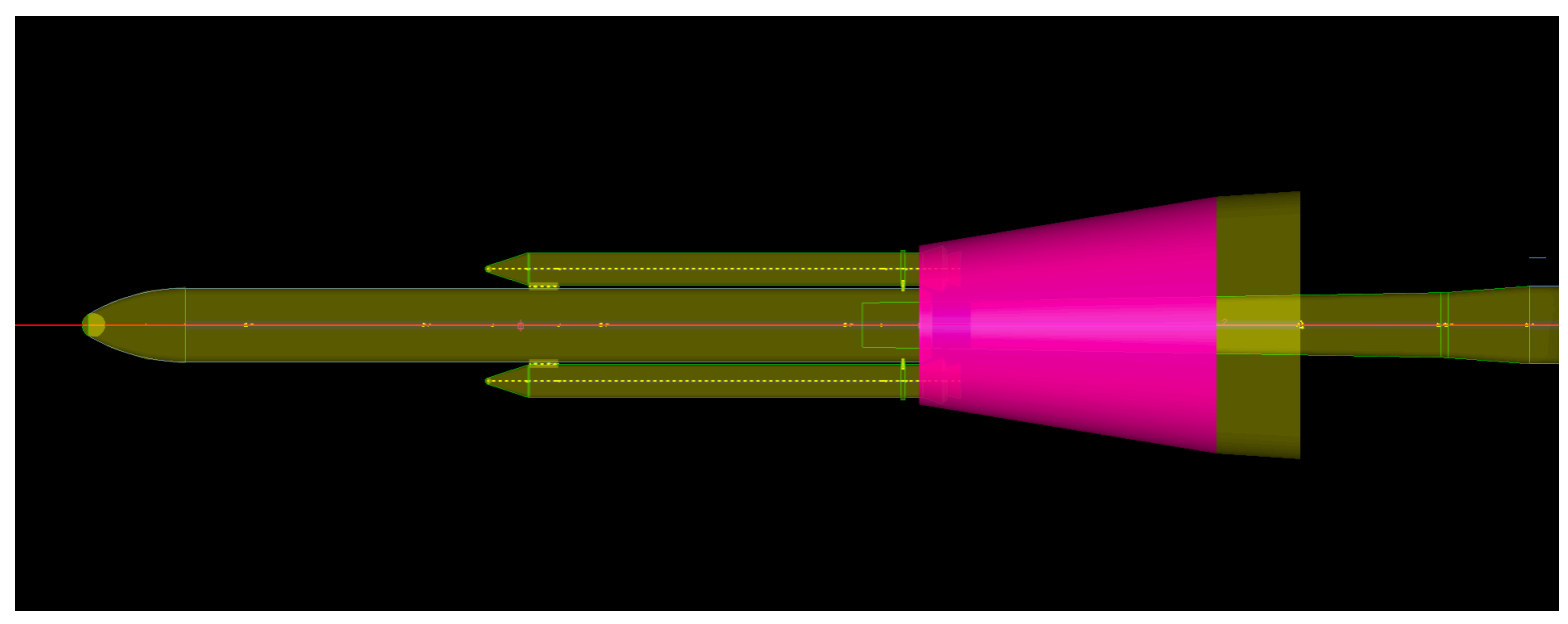

Fig. 4 A side view of the structure of sources in GTC, with the wake volume source highlighted.

from the body in order to adequately resolve the eddy content of the wakes. However, the volume sourcing must be restricted to the wake region as much as possible, since refining regions of the flow where no action is taking place increases the resource requirements of the simulation without providing any benefit. In this study, a sizable volume behind the base of the SLS body was refined with a constant grid spacing to ensure sufficient resolution of the wake.

The flow domain was set as approximately a cube, with far field boundaries located ten SLS-lengths away from the vehicle. The vehicle body is located in the center of the domain and the sting extends from the vehicle all the way back to the outflow boundary of the domain.

Output from GridTool is imported to Heldenmesh, which employs a three-step process to generate an unstructured volume mesh composed of tetrahedra. The first step generates a surface triangulation, the local resolution of which is controlled by the source specification. The second step employs an advancing layer method to grow a boundary layer grid normal to the surface. The third step employs an advancing front method to discretize the inviscid flow region. One of the more beneficial features of Heldenmesh is that in generating the volume grids, the code checks the validity and quality of each tetrahedral cell as it is generated, while ensuring that no holes are left in the grid as the procedure extends out into the far field.

Heldenmesh receives the grid geometry and patch topology in a single file from GTC, then takes a small input file of its own to define variables such as the height of the first viscous layer, the growth rate of successive layers, and the rate at which the volume grid becomes less refined with distance. The sources may be specified in either GTC or Heldenmesh; for this study, sources were specified in GTC. Grids were output as unstructured tetrahedral mesh files. Other outputs include a file mapping the boundary conditions and a file defining which surfaces make up each named component.

For Kestrel, the *.cogsg grid file must be converted to a *.avm file. This was done with the tool Carpenter, which comes with the Kestrel suite of tools. Carpenter also allows one to convert a tetrahedral grid into a mixed element grid, taking most of the boundary layer tetrahedral cells and converting them into prisms, leaving the tetrahedra in the far field grid alone, and combining the remaining cells between the the prisms and tetrahedra into pyramids. Both tetrahedral and mixed element grids were developed for Kestrel. FUN3D is also able to use mixed element grids, but that option was not pursued due to an oversight.

\section{Results}

Preliminary runs were computed with the three CFD codes in order to define suitable procedures for the base force problem. It was found that the RANS solutions from USM3D, FUN3D, and Kestrel all matched each other reasonably well. Each code produced similar features in the afterbody pressure distributions and similar force levels, even with some differences in grids and settings. DDES results from FUN3D and Kestrel matched as well, although those solutions were more sensitive to variations. For instance, Kestrel solutions using mixed element grids exhibited some notable discrepancies from FUN3D solutions for fully tetrahedral grids. However, Kestrel solutions using fully tetrahedral grids matched the FUN3D results nicely. Since it has been well documented that mixed element grids 
are to be preferred over tetrahedral grids for most problems, the oversight in not generating mixed element grids for FUND3D will be addressed in subsequent investigations.

Grid refinement studies were performed for both RANS and DDES to ensure that the solutions were grid independent. A time step study was also performed for DDES to ensure that the simulations captured the correct time scale for this case; a time step of 1 millisecond was deemed sufficient and was used for all further runs. A short, additional study was performed to determine the most suitable extent of the refined grid in the region of the wake at the base of the vehicle. The volume sources used to control the refinement in the wake region were extended 1,2,3, and 4 nominal core diameters behind the model; it was determined that the sources needed to extend at least 3 core diameters back to produce an accurate solution.

Regarding CFD methodology, this study confirmed that RANS CFD is insufficient for detailed analysis of the separated base flow for three-body launch vehicles. Since RANS was already known to be inaccurate when solving highly separated flows, it is no surprise that time-accurate DDES was necessary for a study of this nature. Generally, pressure distributions on the SLS base from RANS solutions were much less uniform than those from DDES solutions.

\section{A. Run Matrix}

The test matrix for this study is defined by three variables: Mach number, sting size, and flow angle. The Mach numbers tested were $M=0.8,1.3,2.0$, and 2.5. These values correspond to test conditions recorded in the wind tunnel experiments and provide both transonic and supersonic cases. The six sting shapes (including the no sting case) are described in Sec. II.A. The flow angles tested were either pure pitch $\left(\alpha=2^{\circ}\right.$ or $\left.4^{\circ}\right)$ or pure sideslip $\left(\beta=2^{\circ}\right.$ or $\left.4^{\circ}\right)$.

This matrix contains 96 cases. While the goal was to test all combinations of these three variables for all three codes, the full matrix was not run with every solver. Instead, FUN3D with the SA turbulence model and DDES was the only method for which the full matrix was completed. The other codes were run on select sections of the matrix for the purposes of comparison and validation. The following results are all from those FUN3D runs.

\section{B. Comparison of Experimental and CFD Pressure Probe Measurements}

Plots of the measured pressure coefficient values from the pressure probes of the AUPWT test and from the CFD emulated pressure probes for the six stings are shown in Fig. 5 for Mach numbers of 1.3, 2.0, and 2.5 at an orientation of $\alpha=2^{\circ}$ and $\beta=0^{\circ}$. For reference, the diagram in Fig. 5(d) indicates the locations where the base pressure probes were installed in the wind tunnel; the photograph in Fig. 3 is also available. One base probe is installed at the centerline of each SRB, and the four core base pressure probes are installed at $90^{\circ}$ increments, offset by $45^{\circ}$ from the $y$ and $z$ axes. Referenced to Fig. 5(d), probe CORE1 is oriented at radial station 2, CORE2 at radial station 4, CORE3 at radial station 6, and CORE4 at radial station 8. The setup for the emulated probes from CFD is discussed in Sec. II.B. The aerodynamic convention for plotting the pressure coefficient values is employed, with the vertical axis direction reversed from the usual orientation; larger values represent more negative coefficient values, i.e., greater suction. Due to the sensitivity of the data, ordinate values of the pressure coefficient are not shown. However, as a guide to understanding the differences, consider that the maximum range observed for any individual probe is on the order of 0.1 .

The suitability of the CFD simulations for assessing sting effects on the base force corrections hinges upon the degree to which the FUN3D results for the AUPWT sting correlate with the values and trends for the AUPWT wind tunnel test. As a start towards discerning those trends, consider just the wind tunnel pressure probe data for the AUPWT test (black squares). It is evident that at Mach 1.3, there is some variance in the pressure coefficient values for the four probes on the core. As Mach number is increased, the magnitude of the pressure coefficient values on the core decrease, as does the variance between the values for the four probes. For the SRBs, the coefficient values are significantly more negative than for the core at Mach 1.3, about the same at Mach 2.0, and slightly more negative at Mach 2.5; the differences in the magnitude of the core probes and the SRB probes as the Mach number changes presents a rather unusual trend.

Next, consider the CFD pressure coefficient values for the AUPWT sting (purple circles). It is evident that at Mach 1.3, there is some variance in the pressure coefficient values for the four probes on the core and as Mach number is increased, the magnitude of pressure coefficient values on the core decrease, as does the variance between the values for the four probes. Comparing those CFD values against the wind tunnel data for AUPWT test, the CFD values consistently underpredict the magnitude of pressure coefficient values, although differences between the CFD and wind tunnel values decrease with increasing Mach number. For the SRBs, the CFD coefficient values are more negative than the CFD core values at all Mach numbers; the CFD significantly underpredicts the SRB pressure coefficient values from the wind tunnel at Mach 1.3, are about the same as the wind tunnel values at Mach 2.0, and are slightly more 


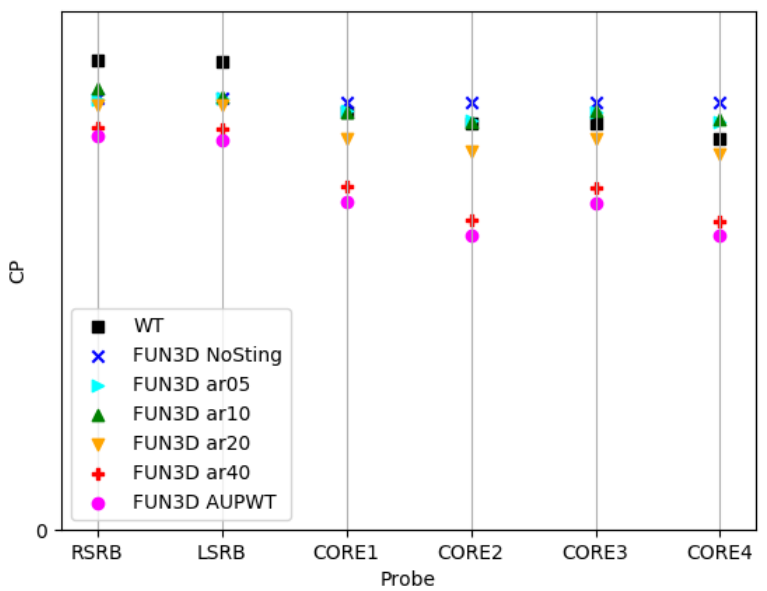

(a) Mach 1.3.

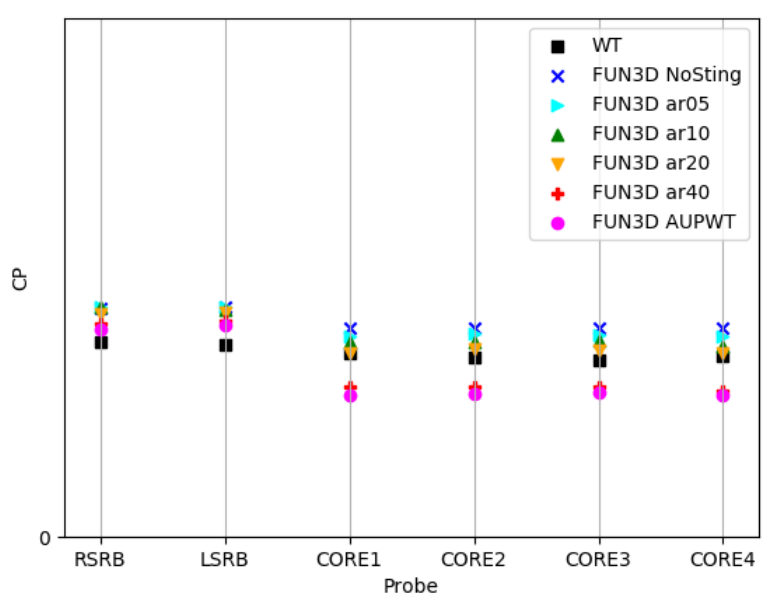

(c) Mach 2.5.

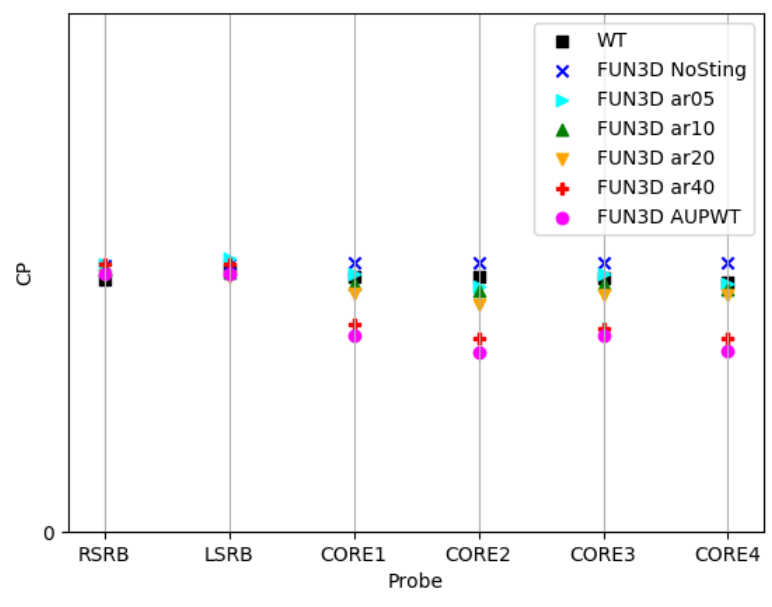

(b) Mach 2.0.

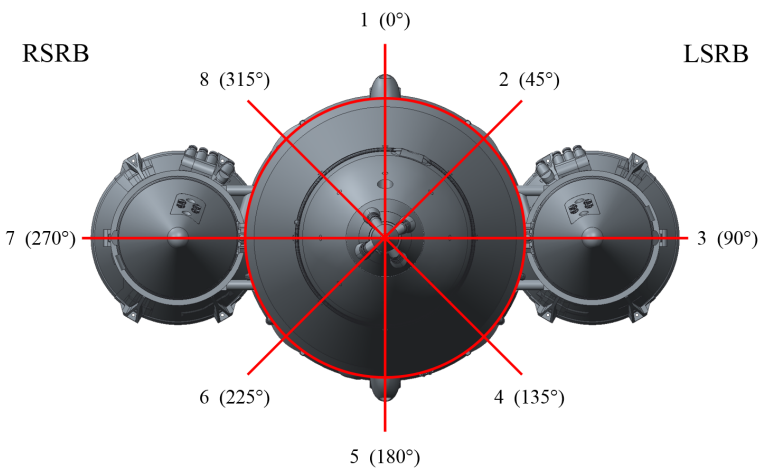

(d) Radial stations used for the AUPWT test (forward looking aft).

Fig. 5 A comparison of emulated CFD base probe pressures for all simulated stings to wind tunnel probe pressures from the AUPWT test at $\alpha=2^{\circ}$ and $\beta=0^{\circ}$. ( $C_{P}$ axes are inverted.)

negative at Mach 2.5.

Considering the CFD results for the other sting configurations, it is evident that as the area ratio is decreased the pressure probe values become more negative; this is the expected trend, since the smaller the area of the sting the greater the size of the wake and, therefore, the greater the amount of suction acting on the base. The fact that the pressure coefficient values for the SRB probes exhibit the same trends exhibited by the core probes indicates the extent to which the sting area sets up the base flow for the entire aft region. It is also apparent that a smaller sting results in a smaller variation between the SRB base pressure measurements and the core base pressure measurements. All cases with a sting appear to track the relative differences between probes (i.e., the shape of the scatter plot) in a manner similar to the wind tunnel data. However, the emulated base pressure probe measurements from CFD simulations with the AUWPT sting correlate the worst with the AUPWT data itself, particularly for the core base pressures, where the magnitude of the suction is consistently underpredicted.

Despite the discrepancies between the pressure coefficient values from the AUPWT test and the values from the CFD for the AUPWT sting, overall, the trends in the two datasets are fairly consistent; for both datasets the magnitudes of the coefficient values for both the core and SRBs decrease with increasing Mach number and the variation in the values on the four probes on the core decreases with increasing Mach number. The differences in trends, namely, that there is more variance in the CFD values for the four probes on the core and that the CFD coefficient values on the 
SRBs are consistently higher than the CFD values on the core, are of concern. Hence, while it is conjectured that the CFD results provide a reasonable representation of the base pressure environment, additional work to characterize the actual environment, including inclusion of the cavity around the sting in the CFD simulations, is required to confirm that assumption.

\section{Afterbody Pressure Distributions}

The full range of Mach numbers and sting geometries was run in FUN3D at flow angles of $\alpha=2^{\circ}$ and $\beta=2^{\circ}$. Pressure coefficient contours on the base of the vehicle are shown at $M=0.8$ and 1.3 , for $\alpha=2^{\circ}$ and $\beta=0^{\circ}$, in Figs. 6 and 7, respectively. A similar set of base pressure contours at $\alpha=0^{\circ}$ and $\beta=2^{\circ}$ are shown in Figs. 8 and 9 . These contours clearly show that larger sting geometries mitigate the low pressure regions created by the separated wake, which was expected. They also hint at the effects that a three-body configuration has on base pressure distributions: pressure on the core base always decreases with proximity to the SRBs. This suggests that the inclusion of the SRBs will decrease the pressure uniformity on the base of the core. This potentially has a negative effect on the accuracy of the base force correction method used in the wind tunnel tests, which utilizes pressure probes in the cavity and not the aft face. The base area on the wind tunnel models is made as small as possible to minimize the base pressure effects, but a base force correction is still applied over these areas assuming a constant pressure equal to the cavity pressure. For simulations at $\beta=2^{\circ}$, the same trend can be seen.

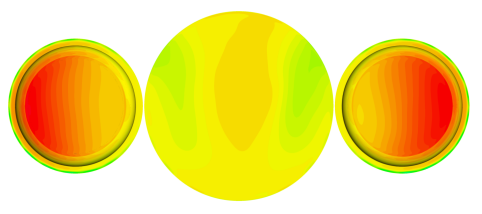

(a) No Sting

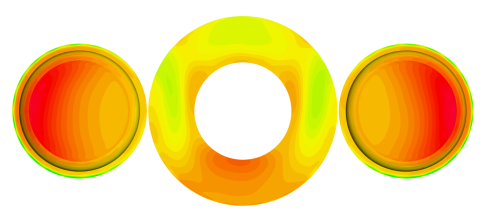

(d) Area Ratio $=20 \%$

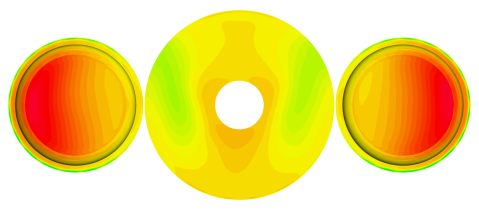

(b) Area Ratio $=5 \%$

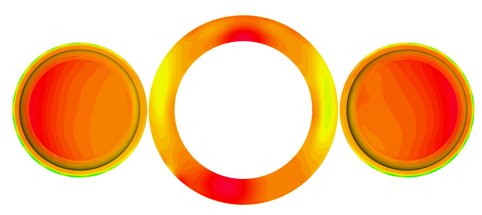

(e) Area Ratio $=40 \%$

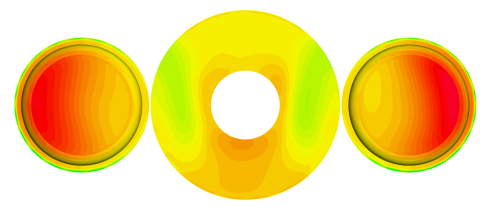

(c) Area Ratio $=10 \%$

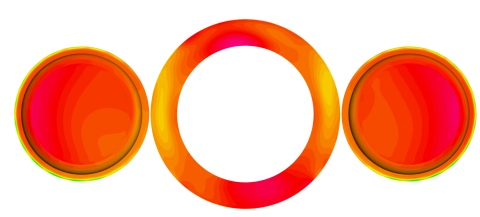

(f) AUPWT Sting

$C P$

Fig. 6 Pressure distributions across the base of the SLS model at $M=0.8, \alpha=2^{\circ}$, and $\beta=0^{\circ}$. Scales are hidden, but constant for this set of figures. The range of pressure coefficients shown are all negative. 


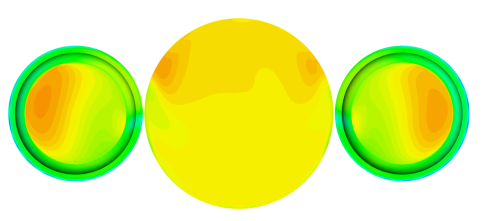

(a) No Sting

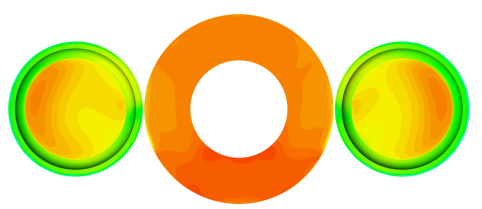

(d) Area Ratio $=20 \%$

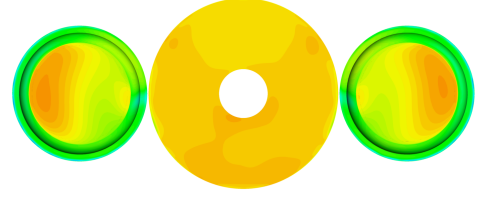

(b) Area Ratio $=5 \%$

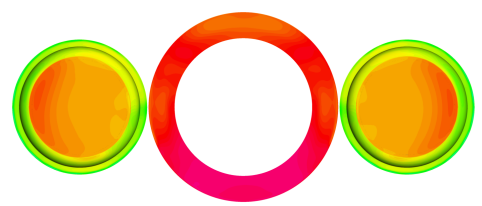

(e) Area Ratio $=40 \%$

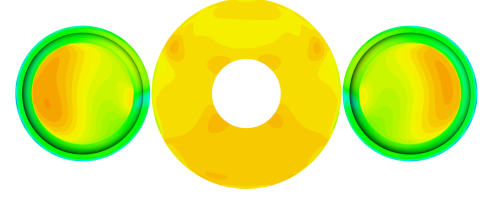

(c) Area Ratio $=10 \%$

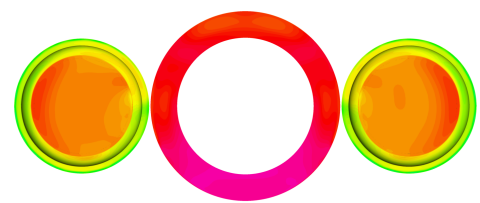

(f) AUPWT Sting

$C P$

Fig. 7 Pressure distributions across the base of the SLS model at $M=1.3, \alpha=2^{\circ}$, and $\beta=0^{\circ}$. Scales are hidden, but constant for this set of figures. The range of pressure coefficients shown are all negative.

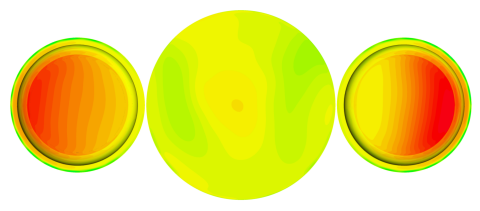

(a) No Sting

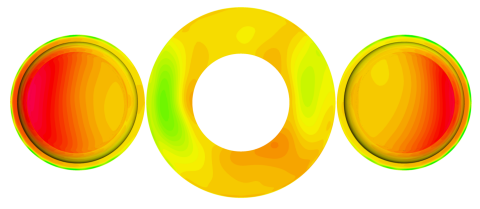

(d) Area Ratio $=20 \%$

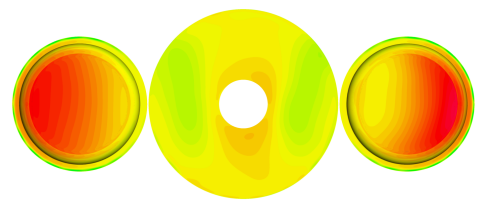

(b) Area Ratio $=5 \%$

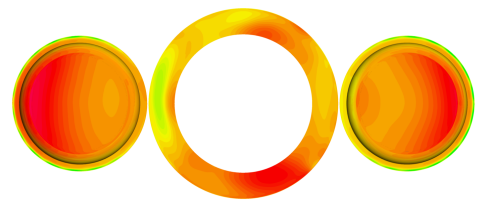

(e) Area Ratio $=40 \%$

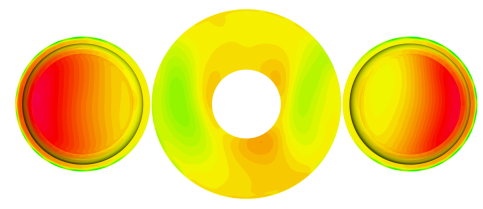

(c) Area Ratio $=10 \%$

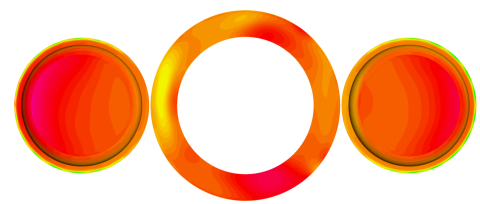

(f) AUPWT Sting

$C P$

Fig. 8 Pressure distributions across the base of the SLS model at $M=0.8, \alpha=0^{\circ}$, and $\beta=2^{\circ}$. Scales are hidden, but constant for this set of figures. The range of pressure coefficients shown are all negative.

The overall effect of sting size on the pressure field upstream of the base can be ascertained from Fig. 10, which shows pressure coefficient distributions around the aft end of the vehicle, on both the surface and a crosscut of the flow field at the $z=0$ plane. Results are shown for the smallest (no sting) and largest (AUPWT sting) stings in the test matrix, thereby representing the most extreme differences. It is evident that, for all three Mach numbers, there is little if any influence of sting size upstream of the back ends of the SRB aft skirts or the boattail of the core. As expected, the flow field downstream of the SRB and core bases experiences a much larger expansion for no sting than for the AUPWT sting, but that effect diminishes with increasing Mach number.

\section{Axial Force Comparisons}

Figure 11 shows the directly integrated forebody axial force plotted against Mach number for all tested sting geometries. What is important to notice about this plot is that the axial force results from all stings nearly match each other, indicating that while the sting size has a dramatic effect on the flow in the separated wake region, it has very little effect on the forces acting on the actual forebody. It is true that there appears to be a slight decrease in forebody axial force as sting size increases, in particular at $M=0.8$ and $M=1.3$. However, the magnitude of that decrease is 


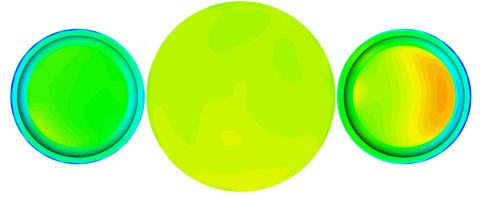

(a) No Sting

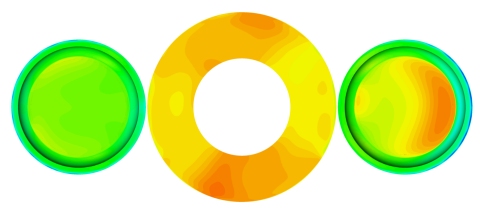

(d) Area Ratio $=20 \%$

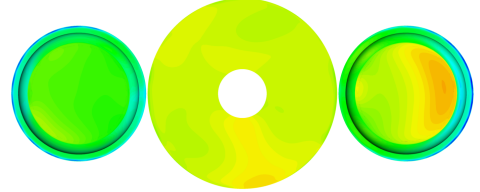

(b) Area Ratio $=5 \%$

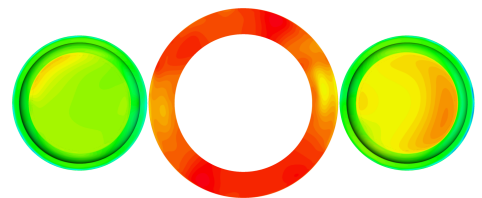

(e) Area Ratio $=40 \%$

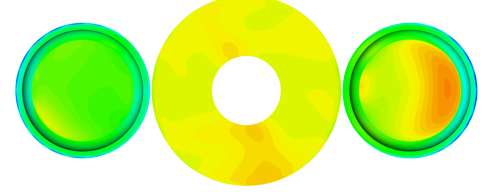

(c) Area Ratio $=10 \%$

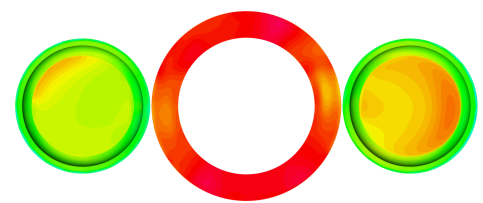

(f) AUPWT Sting

$C P$

Fig. 9 Pressure distributions across the base of the SLS model at $M=1.3, \alpha=0^{\circ}$, and $\beta=2^{\circ}$. Scales are hidden, but constant for this set of figures. The range of pressure coefficients shown are all negative.

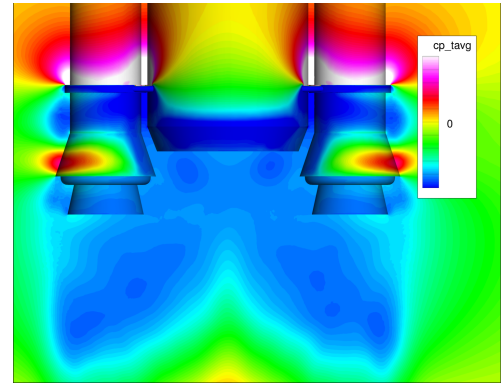

(a) No Sting, $M=0.8$

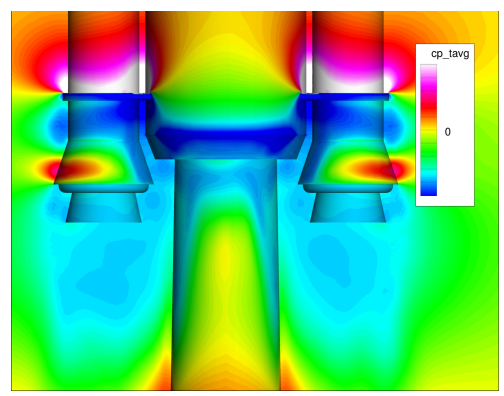

(d) AUPWT Sting, $M=0.8$

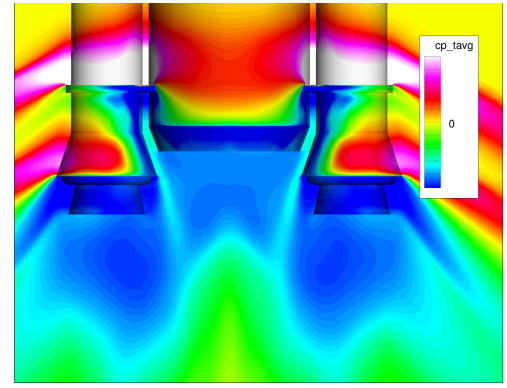

(b) No Sting, $M=1.3$

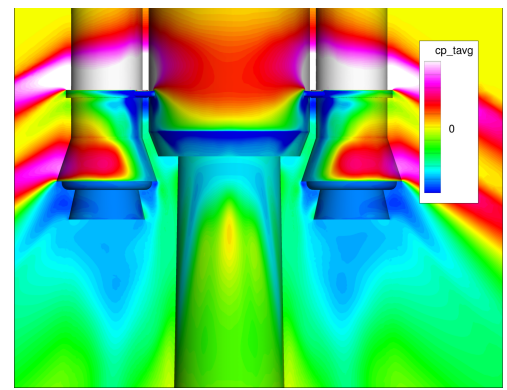

(e) AUPWT Sting, $M=1.3$

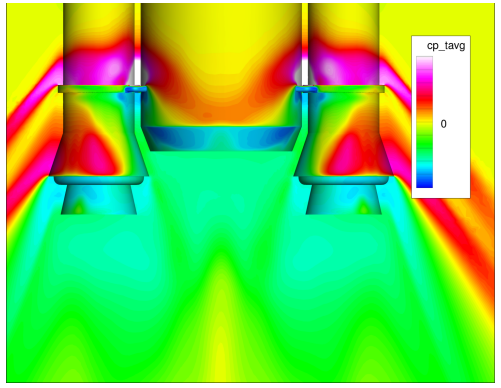

(c) No Sting, $M=2.0$

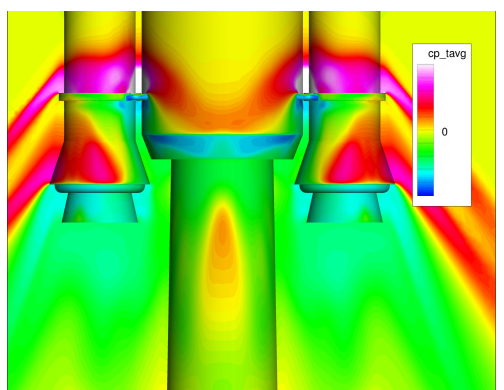

(f) AUPWT Sting, $M=2.0$

Fig. 10 Pressure coefficient distributions around the aft end of the vehicle, on both the surface and a crosscut of the flow field at $\mathrm{z}=\mathbf{0}$, for no sting (top) and the AUPWT sting (bottom), at $\alpha=2^{\circ}$ and $\beta=0^{\circ}$.

within the uncertainty of the data points, so any visible trends can only hint at areas where more detailed work might be warranted.

Applying the emulated wind tunnel corrections to these CFD flow fields yielded several interesting results. First, as shown in Fig. 12, the wind tunnel correction method produces significantly higher forebody axial force coefficient $\left(C_{A}\right)$ values than can be obtained through direct force integration on the forebody. In other words, the wind tunnel methods underestimate the contribution of the base region of the model to the total measured axial force. Examination of the pressure contours shown in Figs. 6-9 reveals that pressure on the base of the core is generally highest in the area directly surrounding the sting. It can also be seen that the pressure at the center of the SRB bases, while not 


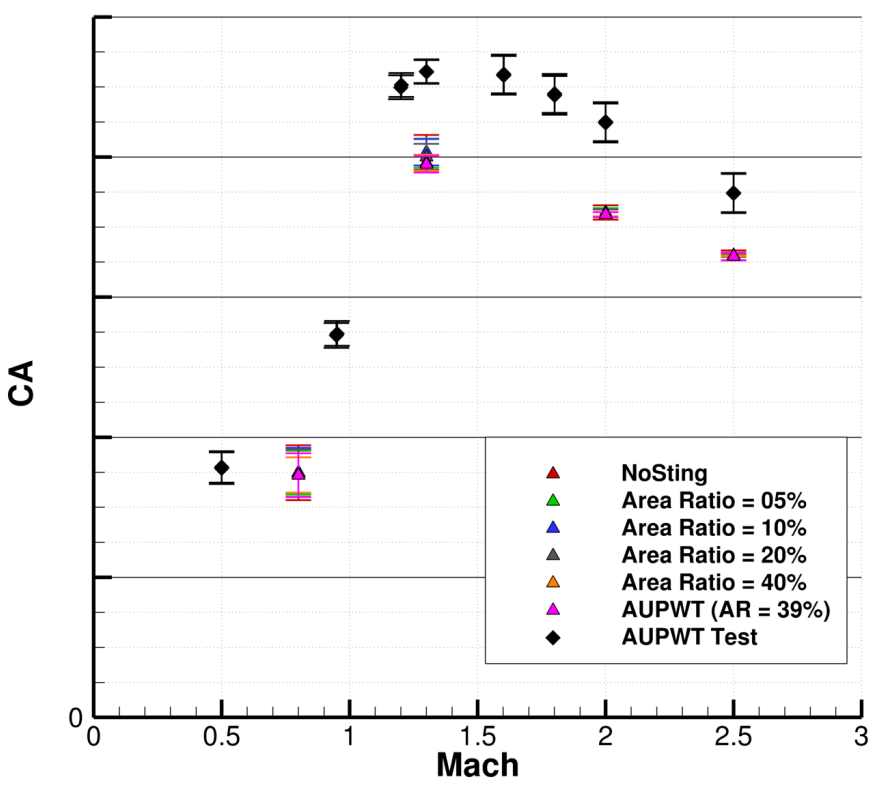

Fig. 11 Forebody axial force coefficient plotted against Mach number at $\alpha=2^{\circ}$ and $\beta=0^{\circ}$. Calculated through direct force integration.

necessarily the point of highest pressure on the bottom face of the SRB, is often much higher than the pressure acting on the SRB skirt base (the outer ring of the SRB bodies, in the base contour plots). These discrepancies result in the pressure probes measuring higher pressure values than the average across the bases of the three bodies, resulting in a lower calculated axial force for the base region than one obtained through direct integration. Since the axial force on the base region is subtracted from the total measured axial force to obtain forebody axial force, this error results in a higher forebody axial force being reported than is actually present.

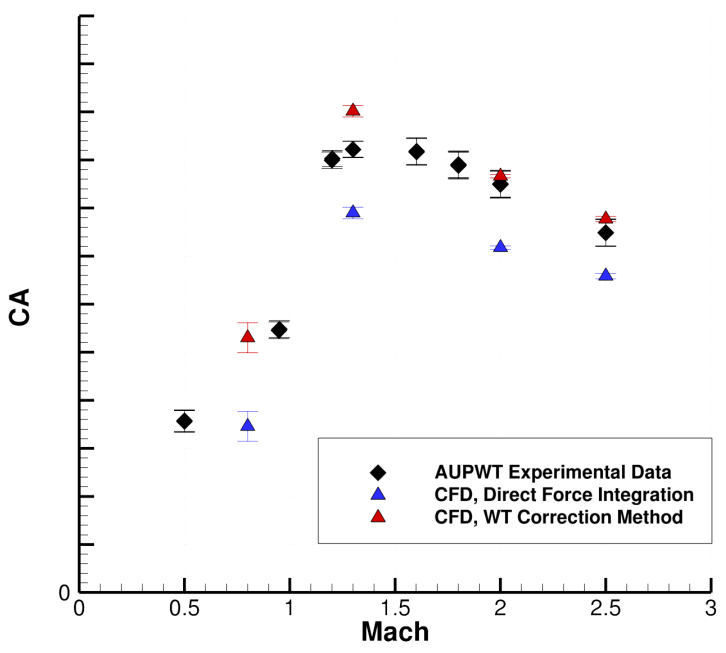

(a) $\alpha=2^{\circ}$ and $\beta=0^{\circ}$

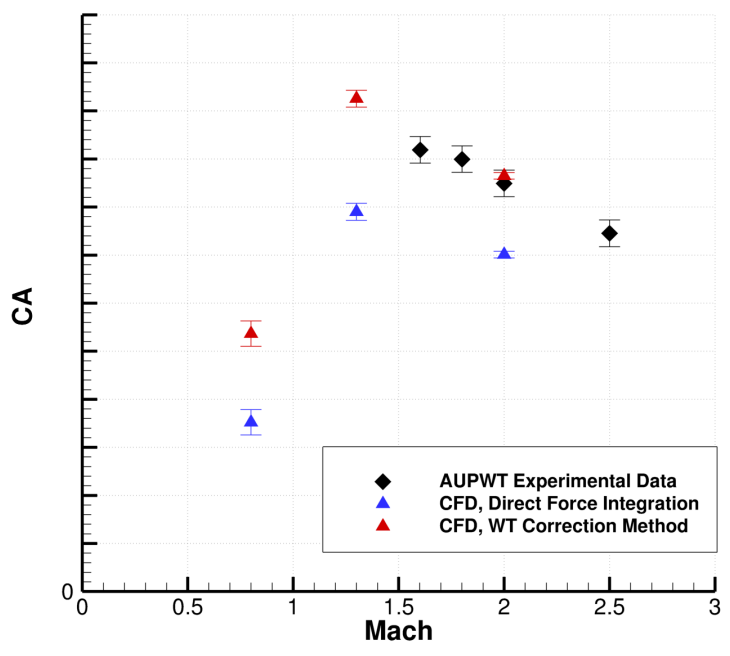

(b) $\alpha=0^{\circ}$ and $\beta=2^{\circ}$

Fig. 12 Axial force coefficient comparison between direct force integration of a FUN3D DDES flow field and an emulation of wind tunnel correction method on that same flow field.

This finding does come with a major caveat: the geometry used for this study does not include the base cavity that is present in the wind tunnel model. It is difficult to predict what effect the cavity has on the base pressure distribution, but it is certainly possible that its inclusion serves to make the pressure more uniform across the base of the core 
and near the four core pressure probes. Additional uncertainty stems from the fact that the areas of multiplication are different between the CFD and wind tunnel models. During wind tunnel method emulation, pressure on the CFD model is multiplied by the area of the core base minus the area of the sting cross section. When processing experimental data, pressure on the wind tunnel model is multiplied by the area of the core base, including the area of the sting cross section. This is done because an open path exists inside the model that allows for the cavity pressure to equalize and act on the entire cavity cross-sectional area. Although both of these operations eventually produce the same output, namely, forebody axial force, it is difficult to know the extent to which the correlation between the two is impacted.

Since the correlation between the CFD and wind tunnel pressure distributions is somewhat unknown, the remainder of this study focuses on the contributions of the SRB bases to the correction error. This should provide a much better direct comparison, since the SRB CFD multiplication areas are calculated in the same way as for the wind tunnel model.

Figure 13 shows the error in the SRB base $C_{A}$ calculations between the wind tunnel correction method and direct force integration. The values are obtained by subtracting axial force calculated using the wind tunnel method from axial force obtained through direct force integration. The different colors of the symbols correspond to the different sting sizes. These plots show that, with the exception of the cases at $M=0.8$, the contribution of the SRB bases to the measured axial force is underestimated by the wind tunnel probe method. However, examining the distribution of the different sting sizes shows that no trend exists between the accuracy of the correction and the size of the sting. In other words, sting size does not influence the uniformity of the pressure on the SRB bases in any predictable way.

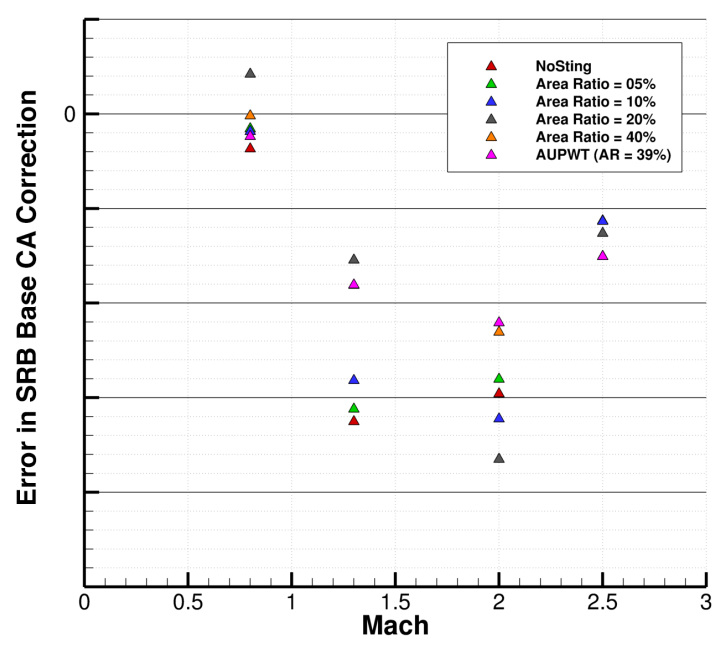

(a) $\alpha=2^{\circ}$ and $\beta=0^{\circ}$

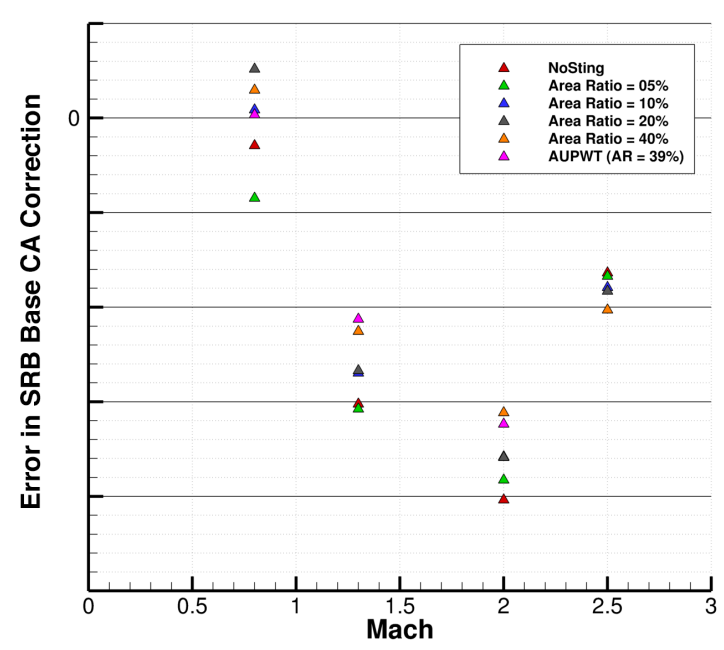

(b) $\alpha=0^{\circ}$ and $\beta=2^{\circ}$

Fig. 13 Error in SRB base $C_{A}$ calculation between the wind tunnel correction method and direct force integration. Each color corresponds to a different sting geometry.

\section{Conclusions}

The RANS and DDES results from this study confirmed that RANS methods are sufficient for simulations of the forebody forces and moments of SLS type vehicles, but DDES methods are required for assessments of the afterbody characteristics. Both RANS and DDES results indicated that changes in sting size have little to no effect on actual forebody axial force when computed with direct integration. Across all Mach numbers, axial force calculated by direct force integration on the forebody changed very little as sting size increased. The slight differences that were present between sting configurations were well within the axial force uncertainty of the solutions.

Even using the DDES results, little could be concluded about the impact of sting size and shape on the accuracy of the SLS wind tunnel axial force correction method. While it is obvious that changes in sting size will change the resulting flow around the vehicle base, it is less clear whether an increase in sting size has a mitigating or exacerbating effect on the correction method error. Part of the reason for the difficulty is the lack of a base cavity on the CFD model, since it is unknown how well the pressure distribution computed on a closed base model matches the pressure distribution present during wind tunnel tests using an open-base model. The CFD solutions do indicate that because 
larger stings reduce the area of the base of the core, they may allow pressure near that surface to be more uniform. A more uniform pressure distribution increases the validity of the pressure probe measurements, allowing for more accurate corrections to be made on the core base. No trend could be found between sting size and pressure uniformity on SRB bases.

The solutions produced by this study did allow some insight into the overall accuracy of the wind tunnel correction method. Axial force on the vehicle afterbody was calculated using both direct force integration and the emulated wind tunnel correction method. The axial force values obtained from the emulated wind tunnel correction matched reasonably well with wind tunnel experimental data. Axial force values obtained by direct force integration on the afterbody were much lower. This would indicate that the wind tunnel correction method used in the experiments tends to overpredict forebody axial force. Again, though, the lack of a base cavity in the CFD geometry probably has a large impact on the pressure distribution near the probes.

The primary deficiency with this investigation is that the cavity surrounding the sting in the experimental setup was not included in the computational model. The primary reason for simplifying the model was because of the difficulty in simulating flow-field regions of near zero velocity for vehicles traveling at transonic and supersonic speeds. As an intermediate step, the next step in this investigation is to computationally model the sting cavity with a porous surface once, of course, an appropriate sized cavity is designed for each of the stings. After that, the full extent of the sting and associated cavity must be modeled.

\section{References}

[1] Pinier, J. T., Erickson, G. E., Paulson, J. W., Tomek, W. G., Bennett, D. W., and Blevins, J. A., "Space Launch System Liftoff and Transition Aerodynamic Characterization in the NASA Langley 14- by 22-Foot Subsonic Wind Tunnel," AIAA Paper 2015-0775, 53 ${ }^{\text {rd }}$ AIAA Aerospace Sciences Mtg., 5-9 January 2015, Kissimmee, FL, USA.

[2] Pinier, J. T., Bennett, D. W., Blevins, J. A., Erickson, G. E., Favaregh, N. M., Houlden, H. P., and Tomek, W. G., "Space Launch System Ascent Static Aerodynamic Database Development," AIAA Paper 2014-1254, 52 ${ }^{\text {nd }}$ AIAA Aerospace Sciences Mtg., 13-17 January 2014, National Harbor, MD, USA.

[3] Shea, P. R., Pinier, J. T., Houlden, H. P., Favaregh, A. L., Hemsch, M. J., Dalle, D. J., Rogers, S. E., Meeroff, J. G., and Lee, H. C., "Ascent Aerodynamic Force and Moment Database Development for the Space Launch System," AIAA Paper, AIAA Aviation 2019 Forum, 17-21 June 2019, Dallas, TX, USA.

[4] Wilcox Jr., F. J., Pinier, J. T., Chan, D. T., and Crosby, W. A., "Space Launch System Booster Separation Aerodynamic Testing in the NASA Langley Unitary Plan Wind Tunnel," AIAA Paper 2016-0796, 54 ${ }^{\text {th }}$ AIAA Aerospace Sciences Mtg., 4-8 January 2016, San Diego, CA, USA.

[5] Cahn, M. S., "An Experimental Investigation Of Sting-Support Effects On Drag And A Comparison With Jet Effects At Transonic Speeds,” NACA-RM-L56F18a, 1956.

[6] Lee, G., and Summers, J. L., "Effects of Sting-Support Interference on the Drag of an Ogive-Cylinder Body With and Without a Boattail at 0.6 to 1.4 Mach Number," NACA-RM-A57I09, 1957.

[7] Love, E. S., “A Summary Of Information On Support Interference At Transonic And Supersoinc Speeds,” NACA-RM-L53k12, 1954.

[8] Tuttle, M. H., and Gloss, B. B., "Support Interference of Wind Tunnel Models - A Selective Annotated Bibliography," NASA Technical Memorandum 81909, 1981.

[9] Frink, N. T., Pirzadeh, S. Z., Parikh, P. C., Pandaya, M. J., and Bhat, M., "The NASA Tetrahedral Unstructured Software System (TetrUSS)," Aeronautical Journal, Vol. 104, No. 1040, 2000, pp. 491-499.

[10] Biedron, R. T., Carlson, J.-R., Derlaga, J. M., Gnoffo, P. A., Hammond, D. P., Jones, W. T., Kleb, B., Lee-Rausch, E. M., Nielsen, E. J., Park, M. A., Rumsey, C. L., Thomas, J. L., and Wood, W. A., “FUN3D Manual: 13.2,” NASA/TM-2017-219661, 2017.

[11] Morton, S. A., McDaniel, D. R., Sears, D. R., Tillman, B., and Tuckey, T. R., "Kestrel - A Fixed Wing Virtual Aircraft Product of the CREATE Program," AIAA 2009-338, 2009.

[12] NASA Langley Research Center, "TetrUSS: Tetrahedral Unstructured Software System, Publications," URL https: //tetruss.larc.nasa.gov/tetruss-related-publications/, Accessed: March 5, 2018. 